\title{
Preservation of splenic vessels during laparoscopic spleen-preserving distal pancreatectomy via lateral approach
}

\author{
Jia-Hui Chen ${ }^{1}$, Kuo-Feng Huang ${ }^{2}$, Chao-Hsu Li ${ }^{1}$ \\ ${ }^{1}$ Division of General Surgery, Department of Surgery, Taipei Tzu Chi General Hospital, Buddhist Tzu Chi Medical Foundation, \\ New Taipei City, Taiwan \\ 2Division of Neurosurgery, Department of Surgery Taipei Tzu Chi Hospital, Buddhist Tzu Chi Medical Foundation, New Taipei City, Taiwan
}

Videosurgery Miniinv 2015; 10 (3): 382-388

DOI: $10.5114 /$ wiitm.2015.54188

\begin{abstract}
Introduction: Preserving splenic vessels during laparoscopic distal pancreatectomy (SPDP-LA) is feasible and avoids unnecessary splenectomy.

Aim: To present our outcomes for this unique technique.

Material and methods: Between January 1998 and January 2012, 6 patients who underwent SPDP-LA for benign or low malignancy tumors in the pancreatic tail were included. Clinical characteristics as well as perioperative data were retrospectively recorded.

Results: All procedures were successful, with an average operative time of $184 \mathrm{~min}$ (range: 88-277 min) and average blood loss of $401.7 \mathrm{ml}$ (range: 10-900 ml). The mean hospital stay was 7 days. Pancreatic fistula occurred in 2 patients but was then cured by external drainage. There was no mortality. Follow-ups were available for all patients. Conclusions: Our experience was characterized by a lack of conversions and by acceptable rates of postoperative fistula and morbidity. The lateral approach showed beneficial results in patients without complications and short post-operative hospital stays.
\end{abstract}

Key words: distal pancreatectomy, lateral approach, spleen preserving, splenic infarction, laparoscopic surgery.

\section{Introduction}

Laparoscopic distal pancreatectomy is considered safe and practical for the treatment of benign or low-grade malignant tumors in the body/tail of the pancreas $[1,2]$. It is associated with a shorter hospital stay and fewer postoperative complications than open distal pancreatectomy [2].

Although laparoscopic distal pancreatectomy can be done with or without preservation of the spleen, spleen vessel preserving distal pancreatectomy (SPDP) is preferred to avoid the risk of overwhelming post-splenectomy sepsis and other complications related to splenectomy [3-5]. Two major spleen-preserv- ing procedures are the Warshaw procedure, which conserves the spleen by blood flow from the short gastric vessels, and the Kimura procedure, which preserves the spleen with splenic vessels $[6,7]$. The spleen vessel preserving procedure is more demanding because it requires meticulous dissection of the pancreas from the splenic vessels. The procedure with division of the splenic vessels is easier but carries a risk of spleen-related morbidity, such as infarction or abscesses due to insufficient splenic blood supply $[6,8,9]$. To reduce this risk, the preservation of an adequate blood supply to the spleen is a priority, and advances in laparoscopic instruments, experience, and good surgical technique ensure that spleen-preserving laparoscopic

\section{Address for correspondence}

Chao-Hsu Li MD, Division of General Surgery, Department of Surgery, Taipei Tzu Chi General Hospital, Buddhist Tzu Chi Medical

Foundation, No. 289, Jianguo Rd., Xindian Dist., 23142 New Taipei City, Taiwan, phone: +886-9-70333650, e-mail: igglee@yahoo.com.tw 
distal pancreatectomy can be performed safely with the preservation of the splenic vessels [8, 10-12].

Some studies have proposed a lateral approach for SPDP (SPDP-LA) and suggested that it may be a safer and easier technique for SPDP $[13,14]$. However, others have disagreed with this view because of certain biases, such as small sample size, imprecise tumor size, the learning curve effect, and selection according to pathology [15].

Herein, we present our experience with SPDP-LA. In this retrospective study, the surgical outcome after SPDP-LA was analyzed and the clinical significance of this tailored approach was evaluated.

\section{Aim}

The aim of the study was to describe the clinical characteristics, indications, technical procedures, and outcomes in laparoscopic SPDP-LA in a single institution.

\section{Material and methods}

All patients who underwent SPDP-LA between January 1998 and January 2012 in a regional hospital were enrolled in this retrospective study. Medical records were retrospectively reviewed, and perioperative clinicopathologic variables, such as gender, age, body mass index (BMI), preoperative physical classification defined as the American Society of Anesthesiologists (ASA) score, pathologic diagnosis, tumor size, operative records, postoperative morbidity and mortality, were evaluated. Pancreatic leak was defined according to the guidelines of the International Study Group on Pancreatic Fistulas (ISGPF) [16].

\section{Position of the patients, surgeons, and trocars}

The patient was placed in a supine position with legs apart. As light anti-Trendelenburg tilt was obtained and if necessary rotated about $30^{\circ}$ to the right. A gastric tube and a bladder tube were inserted. The surgeon stood between the legs of the patient, while the first and second assistant, respectively, stood on the left and right of the surgeon. The scrub nurse was on the right side of the operating surgeon. The monitor was placed over the left shoulder of the patient.

An 11-mm paraumbilical port was inserted using an open method. The intra-abdominal pressure was monitored and maintained at around $12 \mathrm{~mm} \mathrm{Hg}$.
The remaining trocars were placed as follows: 1 in the xyphoid area $(5 \mathrm{~mm}), 1$ in the right subcostal area $(5 \mathrm{~mm}), 1$ in the left side $(12 \mathrm{~mm})$. The $30^{\circ}$ angled scopes were through the umbilical trocar. The surgeon used the instruments placed in the right subcostal area and in the left side (Figure 1).

\section{Exploration of the pancreas}

The greater omentum was divided toward the splenic lower pole while preserving the left gastroepiploic vessels and short gastric vessels. The abdominal surface of the pancreas was exposed and the target lesion was identified through laparoscopic ultrasonography. The retroperitoneum was divided along the inferior margin of the pancreas.

\section{Laparoscopic SPDP-LA}

Separation of the pancreatic gland from the retroperitoneum and the splenic vessels was started from the lateral end of the pancreas toward the pancreatic head. The pancreatic tail was then retracted medially away from the splenic hilum. The dissection then proceeded in a lateral to medial dis-

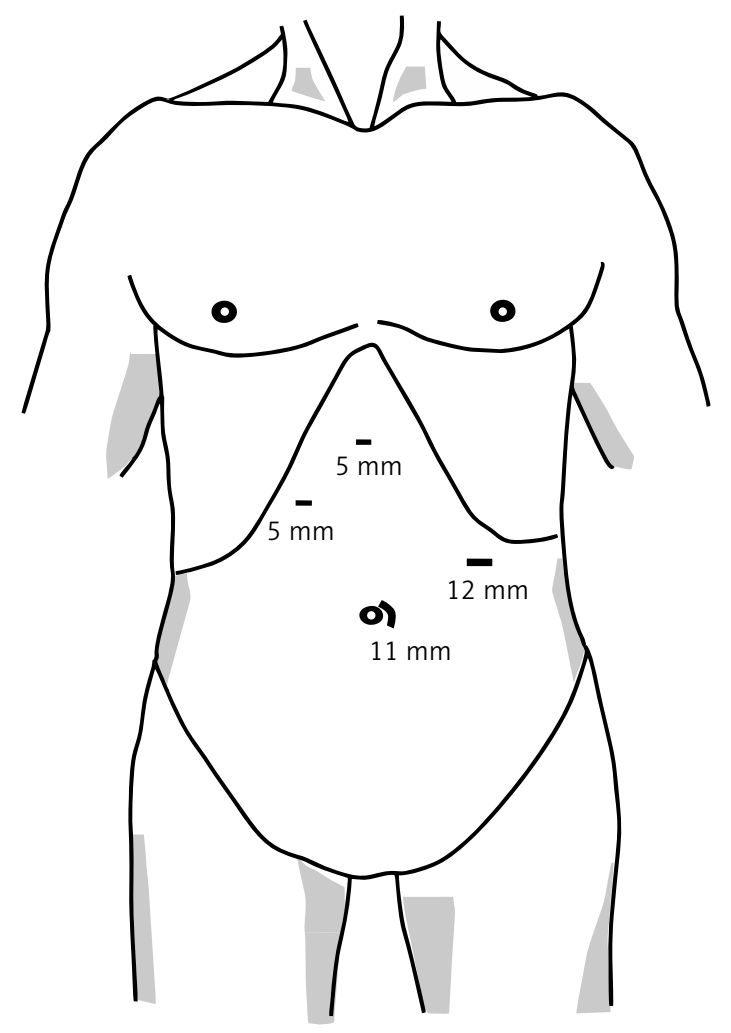

Figure 1. Trocar placement position 
section, ligating each branch of the splenic vessels encountered, using clips or ultrasonic shears (Harmonic scalpel, Ethicon, Cincinnati, USA). After the splenic vein was adequately isolated, the splenic artery was separated from the pancreas using the same method. After adequate surgical margins were achieved, the pancreas was transected proximally to the tumor with one or two 45-mm Endo-GIA staplers (Tyco Healthcare, Norwalk, Connecticut, USA). The type of cartridge (white, $2.5 \mathrm{~mm}$; blue, $3.5 \mathrm{~mm}$ ) was selected according to the thickness and texture of the pancreas. Additional sutures were placed on the pancreatic stump using intracorporeal interrupted polypropylene 2-0 sutures. The specimen was retrieved in a vinyl bag and extracted through a small incision created by extending a port-site incision. A Jackson-Pratt drain was placed near the pancreatic stump.

\section{Results}

\section{Clinicopathologic characteristics}

The clinical and pathologic characteristics of all the patients are shown in Table I. Six patients underwent SPDP-LA including one male and 5 females. The mean age of the patients was 41.3 years (range: 27-61 years) and the mean BMI was $26.5 \mathrm{~kg} / \mathrm{m}^{2}$ (range: $19.1-33.9 \mathrm{~kg} / \mathrm{m}^{2}$ ). Two patients had an ASA score of 3, two had an ASA score of 2, and one had an ASA score of 1 . Pancreatic masses were found incidentally on computed tomography (CT) scan in 1 patient, and were identified during evaluation for chronic abdominal pain in 4 patients and for necrolytic migratory erythema in 1 patient. The final pathological diagnoses of the tumors are detailed in Table I. The mean size of the tumors was $6.5 \mathrm{~cm}$ (range: $2.2-13 \mathrm{~cm}$ ) and the mean length of the resected pancreas was $8.2 \mathrm{~cm}$ (range: $5-14 \mathrm{~cm}$ ).

\section{Perioperative outcomes}

The average operation time was 184 min (range: 88-277 min) with an estimated blood loss of 401.7 $\mathrm{ml}$ (range: 10-900 ml) (Table II). All 6 patients' spleens were successfully preserved and no splenic infarction was noted from follow-up computed tomography. The urethral tube and gastric tube were withdrawn postoperatively. Out-of-bed was allowed at postoperative day 1 . At postoperative day 1, patients had liquid diets, and they had semi-liquid diets on the following days. Once patients took their meals and fluid from the intraoperatively placed drain was less than $15 \mathrm{ml} /$ day, the abdominal drainage tube was withdrawn. The mean postoperative hospital time was 7 days (range: 5-11 days) (Table II). Based on the ISGPF definition, 2 patients had pancreatic fistulas, classified as grade B (33.3\%). The other pancreatectomy-related complication was a pseudocyst ( $n=2,33.3 \%$ ) that was managed with external drainage with resolution. No wound infections requiring antibiotics or wound reopening occurred. There was neither conversion nor mortality.

\section{Discussion}

The traditional surgical approach to the distal pancreas requires large abdominal incisions be-

Table I. Clinicopathologic characteristics

\begin{tabular}{|c|c|c|c|c|c|c|}
\hline \multirow[t]{2}{*}{ Parameter } & \multicolumn{6}{|c|}{ Patient } \\
\hline & 1 & 2 & 3 & 4 & 5 & 6 \\
\hline Age [years] & 33 & 33 & 53 & 41 & 61 & 27 \\
\hline Gender & $\mathrm{F}$ & $\mathrm{F}$ & M & $\mathrm{F}$ & $\mathrm{F}$ & $\mathrm{F}$ \\
\hline BMI $\left[\mathrm{kg} / \mathrm{m}^{2}\right]$ & 26.9 & 33.9 & 22.8 & 19.1 & 27.7 & 28.4 \\
\hline ASA score & $\|$ & $\|$ & III & $\|$ & III & । \\
\hline Pathology & $\begin{array}{c}\text { Benign } \\
\text { non-epithelial } \\
\text { fibroma }\end{array}$ & SPT & Glucagonoma & Pseudocyst & $\begin{array}{c}\text { Serous } \\
\text { cystadenoma }\end{array}$ & SPT \\
\hline Tumor size $[\mathrm{cm}]$ & 8 & 5 & 6 & 5 & 2.2 & 13 \\
\hline Presence of symptoms & Yes & No & Yes & Yes & Yes & Yes \\
\hline
\end{tabular}

SPT - Solid pseudopapillary tumor. 
Table II. Perioperative outcomes

\begin{tabular}{|c|c|c|c|c|c|c|}
\hline \multirow[t]{2}{*}{ Parameter } & \multicolumn{6}{|c|}{ Patient } \\
\hline & 1 & 2 & 3 & 4 & 5 & 6 \\
\hline Operative time [min] & 190 & 195 & 197 & 88 & 157 & 277 \\
\hline Blood loss [ml] & 50 & 250 & 900 & 10 & 450 & 750 \\
\hline Conversion & No & No & No & No & No & No \\
\hline Complication & No & Pseudocyst & No & No & Pseudocyst & No \\
\hline Pancreatic fistula & No & B & No & No & B & No \\
\hline Splenic infarction & No & No & No & No & No & No \\
\hline Hospital stay [day] & 7 & 7 & 7 & 5 & 5 & 11 \\
\hline Mortality & No & No & No & No & No & No \\
\hline
\end{tabular}

cause of the particularly deep position of this gland, and entails possible postoperative complications such as wound infections and incisional hernia, in addition to the obvious functional and cosmetic impairment. Furthermore, the most frequent histotypes of respectable distal pancreatic tumors are currently cystic and endocrine neoplasms, which are often benign and are usually diagnosed incidentally during ultrasound examinations carried out in young women. Therefore, the laparoscopic technique is becoming increasingly popular among surgeons performing distal pancreatectomies. The addition of a spleen-sparing technique also eliminates the theoretical risk of overwhelming post-splenectomy infection and postoperative subphrenic abscess [3-5].

The choice of which specific technique of splenic preservation to use appears to be important in selected patients. Two spleen-sparing distal pancreatectomy techniques have been described. One technique involves varying amounts of dissection of the spleen vein and splenic artery away from the pancreas and division of the small venous branches to the gland; this is known as splenic vessel preservation [7]. The other technique, initially described by Warshaw in 1998, involves careful preservation of the short gastric and splenocolic vessels with distal transaction of the splenic artery and vein before distal pancreatectomy [6]. The spleen maintains its blood supply on short gastric flow. With the Warshaw technique (WT), Adam et al. reported that $10.7 \%$ of patients developed symptomatic splenic infarcts, which required reoperation for splenectomy in around half of these patients (4.7\%) [8]. In a retrospective analysis with long-term follow-up in 158 pa- tients who had undergone open distal pancreatectomy using the WT, the rate of reoperation for splenectomy owing to splenic infarction was $1.9 \%$. Among the 65 patients with postoperative imaging, $23 \%$ had splenic infarcts and $25 \%$ had perigastric varices [17]. The length of hospital stay was significantly shorter in patients who underwent WT, which could be partially explained by the lower occurrence of spleen-related complications associated with WT.

Other complications, especially pancreatic fistula, did not differ between the two techniques. Furthermore, patients with splenic infarcts sometimes required readmission and reoperation for splenectomy, which increased the overall length of hospital stay and affected the cost-effectiveness of the procedure. Regarding the adverse events related to WT, SPDP appears to be the preferable technique. Both techniques are comparable in terms of intraoperative and postoperative morbidity, but the SPDP provided the best chance of conserving the spleen. Adam et al. suggest that SPDP should be attempted when possible and switched to WT in cases of accidental bleeding or difficulties during dissection.

Some studies have proposed that a lateral approach is feasible for SPDP and suggested that it may allow the maximum amount of normal pancreatic tissue to be preserved without additional morbidity [13, 14]. However, some authors have suggested that two main preoperative factors can predict the best approach and possible success for splenic vessel preservation. One was the anatomy of the pancreatic tail, and the other was the presence of inflammation or vessel lamination. They did not agree that the lateral approach is always suitable for 
the splenic vein with a less extraparenchymal course in the tail or patients with intrapancreatic mucinous neoplasia (IPMN) or mucinous cyst [15]. In our study, we have presented our initial experience with SPDP-LA in 6 patients with acceptable outcomes. From the final pathology results, no IPMN or mucinous cyst is noted. Herein, we suggest that SPDP-LA should be performed in selected patients.

Overall, we found that our patients tolerated SPDP-LA well. The present study group compared favorably with other SPDP series in terms of blood loss, length of hospital stay, pancreatic leak rate, and overall complications (Table III). The feasibility and safety of SPDP-LA were also demonstrated by the rate of conversion (0\%) and the rate of reoperation (0\%).

The most widespread technique is prograde pancreatectomy performed by transecting the pancreatic body first and then moving up towards the spleen [18]. Generally, a thin, loose connective tissue occurs between the pancreas and splenic vessels, and dissection along this level can minimize intraoperative bleeding. Our technique consists of a retrograde pancreatectomy with initial mobilization of the pancreatic tail from the splenic hilum and dissection of the distal margin of the gland to look for the splenic vein and artery. As soon as both vessels are identified, it is important to separate them from the parenchyma with an ultrasonic device or clips if needed, going to the proximal resection margin. This is the most risky step because of the relative fragility of the splenic vein branches. Our results in terms of conversion rates compared to laparotomy or to the
Warshaw technique, which was nil compared with conversion rates ranging from 0 to $30.9 \%$ in other cases series [8, 12, 19-21], led us to improve this approach in all patients to prevent intraoperative bleeding and to spare as much pancreatic parenchyma as possible. Other advantages of SPDP-LA include easy reproducibility and the fact that the pancreatic tail is free of the venous groove seen in most patients, which makes it possible to maximize the percentage of spleen vessel preserving procedures while lowering postoperative complications such as splenic infarction and abscess.

Yoon et al. [19] suggest two potential mechanisms which raise the occlusion rate of the splenic vein over that of the splenic artery. First, the splenic vein is so densely adherent to the pancreas that more manipulation is required during dissection of the splenic vein from the pancreas. Second, the splenic vein is susceptible to thrombosis and inflammation due to lack of muscle and elastic fibers, low blood pressure, and a lower speed of blood flow than the artery. Our data showed no vascular obliteration or splenic infarction after SPDP-LA, compatible with a previous report by Hwang et al. after SPDP [10]. Even in their cases with vascular obliteration, its related secondary changes (collaterals and varices) did not cause clinical problems during the follow-up period. Therefore, the high rate of vascular obliteration in conserved splenic vessels and secondary changes in the preserved spleen may not be general phenomena after SPDP and SPDP-LA.

Table III. Results of recent large series of SPDP compared with results in current series of SPDP-LA

\begin{tabular}{|c|c|c|c|c|c|c|c|c|}
\hline Authors/year & $N$ & $\begin{array}{c}\text { Age, } \\
\text { mean } \\
\text { [years] }\end{array}$ & $\begin{array}{c}\text { Blood } \\
\text { loss }[\mathrm{ml}]\end{array}$ & $\begin{array}{l}\text { Operative time } \\
\text { [min] }\end{array}$ & $\begin{array}{l}\text { Hospital } \\
\text { stay [day] }\end{array}$ & $\begin{array}{l}\text { Pancreatic } \\
\text { fistula (\%) }\end{array}$ & $\begin{array}{l}\text { Complication } \\
\text { rate (\%) }\end{array}$ & $\begin{array}{c}\text { Splenic } \\
\text { infarction (\%) }\end{array}$ \\
\hline $\begin{array}{l}\text { Yoon et al./ } \\
2009 \text { [19] }\end{array}$ & 22 & 41 & 300 & 250 & 10 & 31.8 & 68.2 & 18.2 \\
\hline $\begin{array}{l}\text { Baldwin et al./ } \\
2011[20]\end{array}$ & 5 & 81 & 200 & 140 & 4.3 & 20 & 20 & 0 \\
\hline $\begin{array}{l}\text { Zhao et al./ } \\
2012 \text { [12] }\end{array}$ & 21 & 48 & 181.4 & 253.1 & Not listed & 47.6 & 33.3 & 0 \\
\hline $\begin{array}{l}\text { Adam et al./ } \\
2012[8]\end{array}$ & 55 & 52.9 & 342.8 & 214.7 & 8.2 & 16.3 & 27.3 & 0 \\
\hline $\begin{array}{l}\text { Butturini et al./ } \\
2012[21]\end{array}$ & 36 & 47.1 & Not listed & 160 & 8 & 36.1 & 58.3 & 2.8 \\
\hline $\begin{array}{l}\text { Current study/ } \\
2015\end{array}$ & 6 & 41.3 & 401.7 & 184 & 7 & 33.3 & 33.3 & 0 \\
\hline
\end{tabular}


The potential advantage of maintaining perfusion to the spleen in SPDP should be balanced against the possibility of splenic vein occlusion and the potential risk of left-sided portal hypertension [22, 23]. Yoon et al. [19] reported that 9 out of 10 patients with complete splenic vein occlusion after SPDP developed a collateral circulation. However, none experienced gastrointestinal bleeding. In 1 patient in their study, minor collateral vessels associated with total occlusion of the splenic vein before the operation developed into gastric varices. Our patients showed good splenic perfusion from the pre-operative CT, and no patients developed collateral circulation or experienced gastrointestinal bleeding after SPDP-LA, Therefore, the preoperative patency of the splenic vessels should be evaluated carefully when selecting patients for SPDP-LA. If total occlusion of the splenic vessels is evident, an SPDP-LA may not be indicated, because total vascular occlusion may not recover even after resection and left-sided portal hypertension may ensue.

The most common and clinically relevant complication after distal pancreatectomy is a pancreatic fistula (PF), which is associated with complications such as a pancreatic fluid collection, intra-abdominal abscess, wound infection, and sepsis. Although at some high-volume centers, PF after laparoscopic distal pancreatectomy has declined over the past decade, the incidence of PF still rose from $5 \%$ to $30 \%$ $[18,24,25]$. In this study, a large variation in the PF rate was recorded, ranging from $16.3 \%$ to $47.6 \%$ in SPDP and $33.3 \%$ in SPDP-LA. The major reason for the variability may be the lack of uniform criteria for PF. The diagnostic criteria of PF are generally based on clinical signs and laboratory indicators, including the occurrence time, the daily amount of leakage, the duration, leakage amylase, etc. The ISGPF criterion [16] was most frequently used, but it failed to explain whether the amount of drainage was related to the diagnosis of PF.

The original disease, pancreatic transection, pancreas texture, blood supply, and stump closure are factors affecting the incidence of PF. Recently, a BMI $>25 \mathrm{~kg} / \mathrm{m}^{2}$ was also reported as contributing to the increased incidence of PF after distal pancreatectomy [26]. Several authors have proposed different techniques to reduce its incidence. These include various ways of transecting the pancreas (ultrasonic dissector, harmonic scalpel, bipolar cautery, etc.), fibrin glue sealing of the pancreatic stump, and oct- reotide administration. We often used the Endo-GIA staples to close the primary stump of the pancreas. If its texture was hard and thick, we preferred to suture the stump additionally. The total PF rate was $33.3 \%$, and all PF were cured by external drainage. However, the $2 \mathrm{PF}$ patients with a BMI of more than $25 \mathrm{~kg} / \mathrm{m}^{2}$ did not receive additional stump sutures. Herein, we suggest a combination of staples, sutures, and fibrin glue for stump management in patient with high PF factors. Further study of improvements in surgical techniques, including new materials or devices to reduce the $P F$, is required.

It is necessary to interpret the current results carefully, because this study has unavoidable critical limitations. It is basically a retrospective study with a small sample size, so a selection bias must be involved. In the future, perhaps, it will be possible to perform a randomized controlled study able to compare homogeneous populations undergoing SPDP and SPDP-LA.

According to the results from our patients and other previous studies, we consider the lateral approach a safe and feasible procedure, which in expert hands can be completed in a reasonable time and yield good results.

We suggest that SPDP-LA is an acceptable surgical option for most benign and indolent tumors in the body and tail of the pancreas.

\section{Acknowledgments}

We gratefully acknowledge support from project TCRD-TPE-102-37 of Taipei Tzu Chi General Hospital.

\section{Conflict of interest}

The authors declare no conflict of interest.

\section{References}

1. Xie K, Zhu YP, Xu XW, et al. Laparoscopic distal pancreatectomy is as safe and feasible as open procedure: a meta-analysis. World J Gastroenterol 2012; 18: 1959-67.

2. Song KB, Kim SC, Park JB, et al. Single-center experience of laparoscopic left pancreatic resection in 359 consecutive patients: changing the surgical paradigm of left pancreatic resection. Surg Endosc 2011; 25: 3364-72.

3. Holdsworth RJ, Irving AD, Cuschieri A. Postsplenectomy sepsis and its mortality rate: actual versus perceived risks. Br J Surg 1991; 78: 1031-8.

4. Shoup M, Brennan MF, McWhite K, et al. The value of splenic preservation with distal pancreatectomy. Arch Surg 2002; 137: 164-8. 
5. White SA, Sutton CD, Berry DP, et al. Value of splenic preservation during distal pancreatectomy for chronic pancreatitis. $\mathrm{Br}$ J Surg 2000; 87: 124.

6. Warshaw AL. Conservation of the spleen with distal pancreatectomy. Arch Surg 1988; 123: 550-3.

7. Kimura W, Inoue T, Futakawa N, et al. Spleen-preserving distal pancreatectomy with conservation of the splenic artery and vein. Surgery 1996; 120: 885-90.

8. Adam JP, Jacquin A, Laurent C, et al. Laparoscopic spleen-preserving distal pancreatectomy: splenic vessel preservation compared with the Warshaw technique. JAMA Surg 2013; 148 246-52.

9. Melotti G, Butturini G, Piccoli M, et al. Laparoscopic distal pancreatectomy: results on a consecutive series of 58 patients. Ann Surg 2007; 246: 77-82.

10. Hwang HK, Chung YE, Kim KA, et al. Revisiting vascular patency after spleen-preserving laparoscopic distal pancreatectomy with conservation of splenic vessels. Surg Endosc 2012; 26: 1765-71.

11. Choi SH, Seo MA, Hwang HK, et al. Is it worthwhile to preserve adult spleen in laparoscopic distal pancreatectomy? Perioperative and patient-reported outcome analysis. Surg Endosc 2012 26: 3149-56.

12. Zhao YP, Du X, Dai MH, et al. Laparoscopic distal pancreatectomy with or without splenectomy: spleen-preservation does not increase morbidity. Hepatobiliary Pancreat Dis Int 2012; 11 536-41.

13. Nakamura M, Nagayoshi $\mathrm{Y}$, Kono H, et al. Lateral approach for laparoscopic splenic vessel-preserving distal pancreatectomy. Surgery 2011; 150: 326-31.

14. Das De S, Kow AW, Liau KH, et al. Novel approach to laparoscopic resection of tumours of the distal pancreas. ANZ J Surg 2009; 79: 288-93.

15. Dokmak S. Medial or lateral approach for laparoscopic splenic vessel-preserving distal pancreatectomy? Surgery 2012; 152: $143-4$.

16. Bassi C, Dervenis C, Butturini G, et al. Postoperative pancreatic fistula: an international study group (ISGPF) definition. Surgery 2005; 138: 8-13.

17. Ferrone CR, Konstantinidis IT, Sahani DV, et al. Twenty-three years of the Warshaw operation for distal pancreatectomy with preservation of the spleen. Ann Surg 2011; 253: 1136-9.

18. Mabrut JY, Fernandez-Cruz L, Azagra JS, et al. Laparoscopic pancreatic resection: results of a multicenter European study of 127 patients. Surgery 2005; 137: 597-605.

19. Yoon YS, Lee KH, Han HS, et al. Patency of splenic vessels after laparoscopic spleen and splenic vessel-preserving distal pancreatectomy. Br J Surg 2009; 96: 633-40.

20. Baldwin KM, Katz SC, Espat NJ, et al. Laparoscopic spleen-preserving distal pancreatectomy in elderly subjects: splenic vessel sacrifice may be associated with a higher rate of splenic infarction. HPB (Oxford) 2011; 13: 621-5.

21. Butturini G, Inama M, Malleo G, et al. Perioperative and longterm results of laparoscopic spleen-preserving distal pancreatectomy with or without splenic vessels conservation: a retrospective analysis. J Surg Oncol 2012; 105: 387-92

22. Turrill FL, Mikkelsen WP. "Sinistral" (left-sided) extrahepatic portal hypertension. Arch Surg 1969; 99: 365-8.
23. Madsen MS, Petersen TH, Sommer H. Segmental portal hypertension. Ann Surg 1986; 204: 72-7.

24. Corcione F, Marzano E, Cuccurullo D, et al. Distal pancreas surgery: outcome for 19 cases managed with a laparoscopic approach. Surg Endosc 2006; 20: 1729-32.

25. Lebedyev A, Zmora O, Kuriansky J, et al. Laparoscopic distal pancreatectomy. Surg Endosc 2004; 18: 1427-30.

26. Seeliger H, Christians S, Angele MK, et al. Risk factors for surgical complications in distal pancreatectomy. Am J Surg 2010; 200: 311-7.

Received: 30.04.2015, accepted: 17.07.2015. 